\title{
Ion Omesco et sa mise en scène d'Othello
}

\section{(2) OpenEdition}

Journals

Édition électronique

URL : http://journals.openedition.org/shakespeare/1282

DOI : $10.4000 /$ shakespeare.1282

ISSN : 2271-6424

Éditeur

Société Française Shakespeare

Édition imprimée

Date de publication : 1 novembre 1994

Pagination : 205-215

\section{Référence électronique}

"Ion Omesco et sa mise en scène d'Othello ", Actes des congrès de la Société française Shakespeare [En ligne], 12 | 1994, mis en ligne le 26 septembre 2013, consulté le 29 avril 2019. URL : http://

journals.openedition.org/shakespeare/1282 ; DOI : 10.4000/shakespeare.1282 
COSMOPOLITISME ET INSULARITÉ 


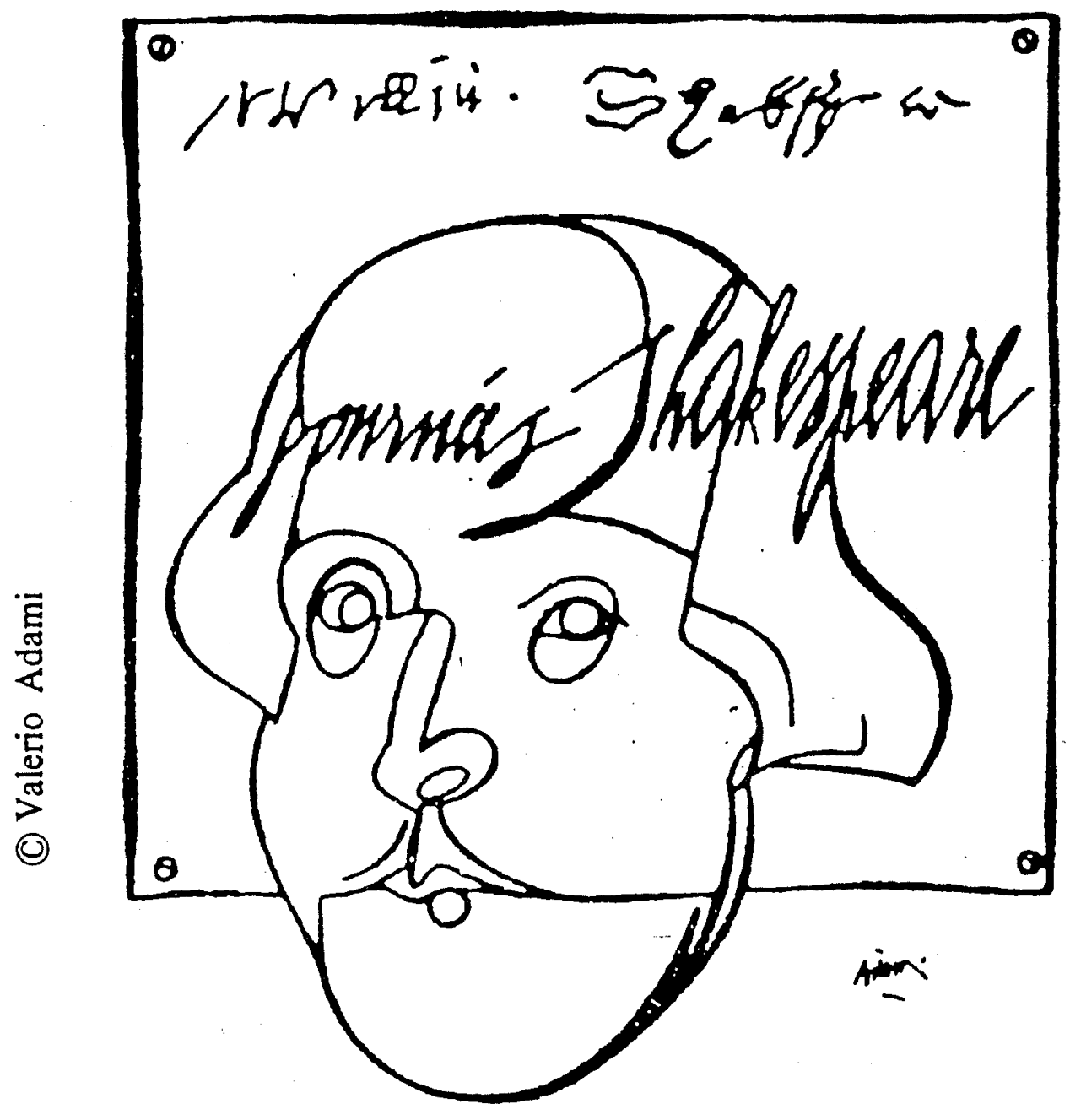

Affiche de Valerio Adami, spécialement conçue pour les journées Shakespeare 1979 


\section{SHAKESPEARE}

COSMOPOLITISME ET INSULARITÉ

Société Française Shakespeare

Actes du Congrès 1993

sous la direction

de

M. T. JONES-DAVIES

Ouvrage publié avec le soutien de

BARCLAYS

BARCLAYS BANK S.A

PARIS

LES BELLES LETTRES

1994 
Tous droits de traduction, de reproduction et d'adaptation réservés pour tous les pays.

(C) 1994 Société d'édition Les Belles Lettres, 95 bd Raspail 75006 Paris

ISBN 2.251.69122-7 


\title{
La mise en scène d'un Othello de chambre
} (fragmentarium)

\author{
Présentation par Dominique Goy-Blanquet
}

Ion Omesco, auteur entre autres d'un ouvrage intitulé Othello, chef-d'cuvre en sursis, publié aux PUF. Scholar and stageman, a mis en scène, la saison passée, un spectacle Othello à Kortrijk, en Belgique.

Cette mise en scène faisait donc suite à un ouvrage où je disais, en commentant l'arrivée du Maure à Chypre, dans l'opéra de Boito / Verdi :

Son armure rutile, le lion de Saint-Marc s'agite sur les bannières, les trompettes célèbrent une double victoire : celle d'Othello contre les Turcs (par forfait), et celle de Verdi sur Shakespeare. Dans la seconde partie du XIXe siècle, les plus grands acteurs, un Rossi, un Salvini, s'offrent des productions qui sont autant d'opéras sans musique. Désormais Othello, chef-d'œuvre en péril, sera pensé en termes de super-spectacle, même par ceux qui n'en ont pas les moyens

(op. cit., p. 52)

Et j'ajoutais :

Mais il est peu probable que l'Othello de Shakespeare puisse trouver dans le gigantisme son visage contemporain. Pour y parvenir il lui faut, je pense, l'intimité qui déconseille l'enflure et rend le pompiérisme impraticable. [...] Plus grand l'espace, plus grand le risque de redondance. Le temps est mûr pour l'Othello de chambre.

(ibid., p. 196)

La salle du Théâtre Antigone, avec son auditorium en amphithéâtre où le comédien pouvait frôler la première rangée des spectateurs, se prêtait à cette vision. 


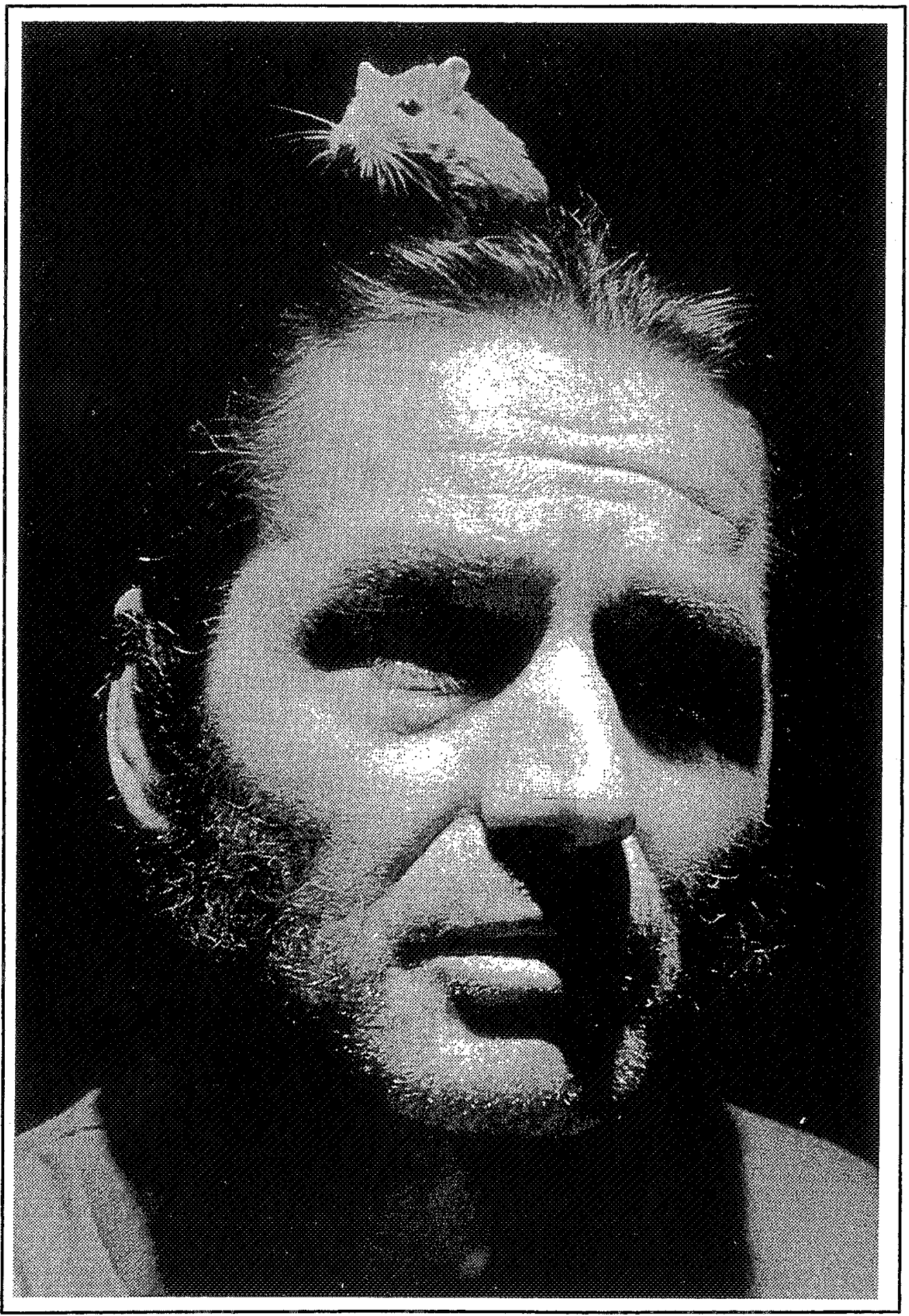

Iago et son confident (Photo : Foto Hol) 
Nous sommes tombés d'accord avec le décorateur Marc Cnops sur un espace scénique entièrement ouvert. Au milieu, un plateau octogonal en cuivre $(\varnothing 5 \mathrm{~m} 75)$; tapis et murs noirs, des étagères pour les accessoires, alternant avec des cubes destinés au repos des comédiens entre les scènes, et des portemanteaux noirs comme le reste.

Un éclairage de base «en douche» délimitait d'une manière rigoureuse l'aire de jeu. Tombée sur le plateau, la lumière faisait vibrer les visages par réfraction, sans tacheter le tapis où se tenaient les comédiens hors jeu, devenus provisoirement des «ombres».

Le spectacle tournait autour de cette action théâtrale, de ce processus par rapport auquel les agissements remplissant la scène, pris un à un, sont des intrigues et des anecdotes : la métamorphose. Non pas celle qui change les yeux d'Alphonso en perles, dans la chanson d'Ariel, ni celle qui transforme le gros roi en festin pour les vers dans l'imagination d'Hamlet. Mais celle non programmée, qui modifie l'ordonnance de la psyché, pour le meilleur ou pour le pire, et rend le personnage méconnaissable. Othello bien sûr, mais également Desdemona, Emilia, Cassio ou Roderigo, assassin inattendu. Pour des raisons différentes, chacun des personnages impliqués d'un bout à l'autre dans cette tragédie pourrait faire sien l'oxymoron planté par lago dès le départ :

I am not what I am

«Je ne suis pas comme j'apparais aux autres», mais aussi «Je ne suis comme je crois l'être» et surtout «Je ne sais pas qui je serai».

Un troisième souci m'habitait : transcrire convenablement en termes de spectacle le paradoxe fondateur de Othello, tragédie à structure comique, dont la trame - une jeune fille amoureuse déjoue la vigilance du père - les procédés (malentendu, imbroglio, écoutes, importance des accessoires), le personnage clef rappellent irrésistiblement ceux de la farce; notamment, de la commedia dell'arte. Il serait superflu d'insister ici sur les similitudes qui lient Iago, ce factotum ingénieux, à l'infatigable Arlequin et, par son côté sombre, à Brighella.

Il fallait, également, assurer la fluidité des changements, passer sans accroc et au plus vite d'une ambiance à l'autre, pour transformer en moments forts la servitude de ces manœuvres. Trois modules prismatiques $(1.80 \mathrm{~m} / 1.20)$ à roulettes firent l'affaire. Les «ombres» rapprochaient ou éloignaient du plateau en cuivre 
les engins dont ils offraient une nouvelle face et, de ce fait, la lumière aidant, les éléments d'une autre ambiance.

Sur l'octogone, un seul objet de taille, à structure métallique. Il apparaissait comme char (II, I), se transformait en table (II, II), en promontoire (III, III), en siège (IV, I), en miroir (IV, II) et finalement en lit (IV, II). On retrouvait dans cette plurifonctionnalité un rappel discret de la métamorphose.

\section{II}

Du point de vue strictement technique (le vilain mot, incontournable), Othello pose au metteur en scène plusieurs cassetête. A savoir: la première intervention de Brabantio, l'approche des monologues de Iago, l'entrée d'Othello et de sa suite, l'apparition tardive du mouchoir. S'ajoutent - difficultés d'un autre ordre - le télescopage dont la scène climactique est faite (III, III), la grotesque dégradation du Maure, la scène du «bordel» et les moments, au dernier acte, où le génie du dramaturge sommeille, tout en faisant beaucoup de bruit. Quandoque dormitat... Comment s'extraire de ces pièges dans un Othello de chambre, centré sur la surprise du détail ? C'est la question.

$\mathrm{Au}$ début du spectacle, les treize membres de la troupe, habillés de robes noires, gantés, le haut du visage caché par un loup, la taille serrée par une ceinture nouée, entouraient le plateau et s'en approchaient jusqu'au moment où le son et l'éclairage atteignaient leur paroxysme. Alors les interprètes reculaient, certains prenaient le chemin des loges, d'autres s'asseyaient sur les cubes. L'hommeorchestre (Guido Desimpelaere), un spectacle dans le spectacle, gagnait sa place derrière son synthétiseur, et le tambour (Ivo Van Der Borght), la sienne. A vue, bien sûr. Le dos au public, l'intrigant et son pigeon jetaient leurs robes à terre - c'était parti.

1. L'entrée d'un père bafoué. Au vers 78, Roderigo (Marc Stroobants) se dirige vers la salle : «Brabantio, signor Brabantio !» et une fenêtre aux contours de lumière apparait sur le cuivre à ses pieds. Echange de gentillesses, Iago (Eddy Brugman) se coiffe d'un couvre-chef à cornes offert par une «ombre» - clin d'œil à son ancêtre - il introduit sa tête entre les jambes de Roderigo pour lancer au sénateur les saletés que Vigny refusa de traduire «par 
respect pour quelques dames qui savent l'anglais». On aurait dû entendre la voix du père, sans le voir (principe non respecté) :

BRAB. - This thou shalt answer, I know thee, Roderigo.

ROD. - Sir, I will answer anything. But I beseech you...

La fenêtre s'éteint, Roderigo s'arrête court, puis une autre fenêtre s'allume au second plan et le jeune homme redémarre ; une troisième, comme si Brabantio (Didier Delmotte) traversait, quelque part en haut, candélabre à la main, une longue galerie, pour se montrer en fin de course de derrière un module. Débraillé (mais surtout pas en chemise de nuit), il est pris en chasse par un serviteur fort pointilleux qui s'applique, entre deux rebuffades, à le rendre convenable. Dans sa belle quarantaine, Brabantio était plus jeune que le Maure.

Ce début donnait le ton; celui d'une mise en scène métonymique où un fragment suggère et remplace le tout.

2. Le mouchoir faisait son apparition dès la scène du sénat (I, II). Une fois le contentieux réglé par un doge (Patrick Rozé) au masque de carnaval, confondant de «vérité», c'est à travers le mouchoir, déployé par Desdemona, qu'Othello lui donnait le premier baiser coram populo. Le combien célèbre accessoire refaisait surface au second acte. Desdemona s'en recouvrait brièvement le visage, sur les falaises de Chypre, dans une attente angoissée (II, I).

Pour marquer par une image visuelle les vertus magiques de l'objet, on a pris un mouchoir d'illusionniste que Iago soulevait d'un souffle après l'avoir subtilisé (III, II, 325).

3. Les monologues du machiavel-metteur en scène. Si Iago parle beaucoup, ce n'est pas seulement par vanité, pour brouiller les cartes à l'instigation de son créateur, ou pour asseoir son ascendant. La logorrhée met son esprit en branle et stimuie ses talents d'improvisateur. Venons en au trois grands monologues: ce Iago peint par lui-même fait des victimes parmi les comédiens. Car ils invitent l'interprète à un satanisme anachronique. Surtout quand, seul en scène, il s'imagine mieux maîtriser de cette façon un grand espace. Et si c'étaient des scènes à deux ?

Nous sommes à la fin du premier acte. Quelque part à l'extérieur, Roderigo broie du noir. Iago passe, aperçoit son homme, le moque, le console et lui tient un laïus sur l'art de réussir sa vie. Remonté, le jeunot s'en va brader ses terres. 
Notre Iago traversait le plateau à toute allure, chargé d'un oiseau en cage ? Celui, sans doute, de Desdemona confiée à ses bons soins. Roderigo parti, il soulevait la housse. C'était un rat. Eddy Brugman lui glissait une friandise entre les barreaux, lui faisait part de ses incertitudes, et son scénario prenait tournure :

I ha't, it is engender'd.

A Chypre, après le départ des autres pour la ville (II, I, 280), le «scélérat» fouille les décharges de sa mémoire pour nourrir sa haine. Le travail du personnage et celui du dramaturge s'entrecroisent.

Iago-Brugman se passait une pommade contre les moustiques. Un bruit léger. Il ouvrait la cage, laissait la bestiole l'explorer et l'attrapait pour lui parler de bouche à oreille. A la fin, il la transvasait soigneusement dans un étui attaché à sa ceinture, lui faisant part de sa trouvaille : il rendra le Maure fou de jalousie. Hein? «this here but yet confus'd».

A l'heure du dernier monologue Iago prenait place sur la table et ouvrait l'étui. Le rat monté sur l'uniforme, sur le cou penché complaisamment, poursuivant son escalade jusqu'au sommet. Parfois il redescendait à point nommé pour entendre son ami lui chuchoter que le final tragique, la catastrophe serait le produit de la bonté.

C'était un rat plutôt mignon qui, au début, n'inspirait pas encore la répugnance de rigueur.

4. Entre Othello et son armée. Combien de mercenaires en armes l'accompagnent-ils? Quinze, vingt ? Ce serait trop et pas assez. Cinq, trente ? Aucun. Prenons nos distances par rapport à la mimesis, pour couper court à toute comparaison avec le film ou l'opéra. Pas de soldats sur le plateau. En revanche, les modules pivotaient, se regroupaient, et sur leur deuxième face apparaissait un collage de têtes casquées. La même tête, multipliée maintes fois et disposée, par réduction, en perspective.

L'apparition de cette armada s'enchaînait avec l'entrée du rôle titre assoupi dans son char, tiré par un clown inquiétant (Kristiaan Lagast), la poitrine couverte de décorations. Une secousse réveillait Othello, héros d'une guerre remportée par la tempête. A ce moment le texte réclame l'extase à deux; pour le couple, le reste du monde a disparu. A cheval sur le protocole, notre Montano entamait son speech de bienvenue à l'aide d'un apo- 
cryphe «mon général !» Othello, pas de réaction. L'autre revenait à la charge - toujours rien. Et Iago d'intervenir. Sa voix, celle du devoir, le ramenait sur terre. Othello effaçait alors l'intermittence du cœur par l'abondance des embrassades. Ce détail, telle l'intrusion d'un thème qui reviendra en force, disait la double faille du protagoniste.

5. Le télescopage de la tentation. Au bout de vingt minutes de temps scénique ininterrompu, durant lequel l'horloge du foyer et celle du drame sont synchronisées, le Maure décide d'en finir une fois pour toutes avec l'objet de son adoration. Il commence par :

Excellent wretch, perdition catch my soul, But I do love thee, and when I love thee not, Chaos is come again (III, III, 84-86).

Et il conclut :

I will withdraw

To furnish me with some swift means of death For the fair devil $[\ldots] \quad$ (III, III, 210-212).

Comment en est-on arrivé là ? L'intervention de l'intrigant n'explique pas tout. Revenons en arrière ; avant même le début de cette longue scène qui passe tellement vite. La jeune fille de Brabantio, devenue femme, veut changer de look. Inexpérimentée mais volontaire, elle s'applique de son mieux à surprendre Othello. A l'épater. Ne sommes-nous pas aux temps où, à Venise, les femmes de tout bord, riches ou pauvres, honnêtes épouses ou courtisanes, se livraient aux pires excès vestimentaires ? Le Maure, hélas, vient d'un pays (lequel ?) où les épouses sont moins émancipées.

Peggy Schepens quittait sa tête nue, son manteau stricte de la veille (conçu par Mircea Marosin), pour un chapeau à plumes et un decolleté profond protégé par un ombrelle. Décidée d'obtenir coûte que coûte la réintégration du charmant Cassio (Vastert Van Aardenne) dans l'armée. Le Maure marquait un moment de stupeur. Sa femme n'était plus sa femme. Il s'approchait, touchait avec appréhension la perruque, la soulevait, et il éclatait de rire. Mais cette perplexité allait rester en attente dans son esprit. Qui plus est, ce changement radical de tenue s'accompagnait d'un nouveau comportement.

En effet, au terme de sa première nuit d'amour, Desdemona se rend compte du pouvoir qu'elle exerce sur son mari. Fort de cet 
ascendant, elle s'immiscera dans ses affaires professionnelles. Qu'on rappelle Cassio ! Cette insistance, faite en la présence de Iago, ce compagnon sans peur et sans reproche, gêne le général.

Peggy tapait de l'ombrelle contre le promontoire et, au moment où Othello voulut s'emparer de ses jumelles, elle les mit hors de sa portée. Ça alors!

I will deny thee nothing,

Whereon I do beseech thee, grant me this,

To leave me but a little to myself (III, III, 8486).

Conseillée tardivement par son instinct, Peggy ôtait sa perruque, se débarrassait de ses chaussures en les projetant en l'air, faisait le salut militaire et décampait à toute vitesse comme un enfant. Mais le mal était fait.

L'«honnête Iago» reprenait les préliminaires de ses travaux de sape commencés au vers 35 , tout en cueillant, un à un, les objets disséminés par la maîtresse. Au moment $\mathrm{M}$, au diable vat !

She did deceive her father, marrying you ;

And when she seem'd to shake and fear your looks,

She lov'd them most

(III, III, 210-212).

Pour accompagner son aveuglante pseudo-preuve par la logistique du visuel, l'inconsolable personnage laissait traîner ses doigts parmi les plumes. Quelle mystificatrice!

6. Le passage du quiproquo (IV, I, 92-165) met le protagoniste dans une position qui risque de lui être théâtralement fatale. Que faire, par la suite, pour remonter la pente ? Bref, Iago lui conseille de se dissimuler quelque part pour écouter les commentaires de Cassio. "Aussitôt dit que fait, un quiproquo classique. Iago pose à voix basse des questions sur Bianca (Mitta Van Der Maat), l'ex-lieutenant s'esclaffe, Othello comprend tout de travers et s'imagine qu'il s'agit de sa femme [...] le capitaine-général se mue en écouteur aux portes, la tragédie, en farce grinçante». Et ces commentaires qu'il délivre, comme le plus minable des cocus !

Autant pousser la farce jusqu'au bout. Dans notre spectacle, Othello, le dos au public, prenait quelque chose des mains de Iago, et se dissimulait derrière le siège avancé pour l'émissaire du doge. Ensuite à la réplique de Cassio.

I marry her? What ? a customer ; 
sa tête faisait irruption au-dessus du siège, munie du nez phallique de Pantalone. Bert André se montrait à trois reprises sans proférer un mot.

7. La scène du «bordel » vient après une suite de cruautés qu'Othello subit et fait subir. Nous savons qu'il ne peut vivre ni avec ni sans ce diable blanc, sa femme. Il est terrifiant et familier. $\mathrm{Sa}$ «folie» rencontre secrètement la nôtre agrandie au microscope. Que dire de Desdemona ? Admirable, mais. Partie avec son prince exotique, balayant son père de sa mémoire - cette Imogène transformée ultérieurement en Ophélie, apparaît plus énigmatique. Plein feux sur elle! Au-delà du volontarisme de Desdemona I et de l'angélisme saint-sulpicien de la seconde, faisons briller à la faveur d'un geste choc la passion qui couve sous la bienséance, se nourrissant d'injures et de coups.

Donc, assis sans gêne sur la table basse, Othello interrogeait Emilia (Mieke Verheyden) sur le comportement de sa femme. Celle-ci s'approchait, embrassait ses genoux et s'entendait dire qu'elle est fausse comme l'enfer.

To whom, my lord, with whom? How am I false?

Le Maure sanglotait, il ne voulait plus la tuer. Qu'elle s'en aille!

away! away! away!

Au moment où Othello-André se levait pour partir, Desdemona lui sautait sur le dos comme une bête aux abois. Pendant la tirade qui s'ensuivait elle restait désespérément agrippée à lui.

8. La dernière scène. Othello, qui se veut prêtre sacrificateur, sera juge en sa propre cause, procureur et bourreau, dans la plus grande confusion des rôles. Othello-André entrait dans une robe longue à motifs qui rappelait ses origines, et il recevait son flaming minister de la part d'une «ombre». Peggy se réveillait, finissait par comprendre, se blottissait à la tête du lit incliné à trente degrés, redescendait, repoussait son agresseur de toutes ses forces, s'échappait. Othello l'attrapait dans un saut, et l'étouffait au premier plan dans les coussins. A la porte, imaginaire, les coups d'Emilia s'amplifiaient.

Le Maure cachait sa victime à l'aide d'un tapis et la ramenait vers lui. Le fait d'avoir un décor ouvert, sans murs ni portes, épargnait maints déplacements pénibles à ce moment. Il suffisait 
de dire «entre, Emilia» et c'était fait. Elle apercevait une main qui dépassait, découvrait sa maîtresse, et Desdemona retrouvait sa voix pour disculper son assassin. Sa résurrection, tout aussi insupportable que le fut sa mort, mais pour d'autres raisons, se passe de commentaires. Nous avons adopté sur ce point l'interprétation de Zoë Wanamaker qui, dans le spectacle de la RSC de 1989, lisait à haute voix les mots inaudibles se formant sur les lèvres de la mourante.

Enter Montano, Gratiano, Iago and others, dit le texte. Ensuite, Emilia fait triompher la vérité. Après son admirable tour de force, elle meurt en odeur de sainteté et d'eau de rose, poignardée par son mari qui prend la fuite. Engagée sur la voie qui mène au degré zéro du mouvement, Othello-André se tenait sur son coussin et serrait Desdemona contre lui, prêt à bondir pour la défendre. Comme si son cadavre lui était encore plus précieux que ne lui avait été son corps vivant.

C'est ensuite que les choses se gâtent. La tragédie perd sa grandeur dans la grandiloquence et dans la platitude des précisions. A partir de là, une mise en scène sans coupures - or nous les fîmes - tourne au calvaire. Notre Othello contournait le débordement imprécatoire, mettait sa victime sur le lit en pente et cueillait, çà et là, ces mots de rien du tout où se rencontrent l'insupportable et la poésie : «cold, cold, my girl, / Even like thy chastity» (V, II, 275-276). Avec l'entrée de Lodovico, de Cassio, et la réapparition de Iago en prisonnier, tout est en place pour la fin. Othello rate sa vengeance. Il entame alors son dernier discours dans la plus grande simplicité :

Soft you, a word or two ...

Puis il la quitte, se poignarde ; et il expire dans un euphuisme :

I kiss'd thee ere I kill'd thee, no way but this,

Killing myself, to die upon a kiss.

Nous avons laissé le dernier mot à Cassio, appuyé sur ses béquilles, lui confiant aussi la dernière réplique d'un Gratiano $a b-$ sent : «All that's spoke is marr'd». Deux «ombres» - l'une côté cour et l'autre côté jardin - avançaient sur le plateau, portant chacune une moitié de cage. Elle se refermait sur Iago, l'emprisonnant jusqu'au cou. Ensuite les «ombres» et les autres personnages se retiraient, la lumière baissait, et sur la troisième face des modules, aussi loin que possible, s'allumaient des constellations. Acces- 
soires du spectacle théâtral, les étoiles prenaient la place tenue par le mouchoir.

Dominique Goy-Blanquet me fait remarquer, qu'il y a un autre Iago en cage, dans l'Othello d'Orson Wells. Nous l'avons tous vu. Je mets en avant la différence de traitement. L'épisode du film frôlait le naturalisme, l'autre, le rituel. Si licet componere...

\section{NOTES}

La première du spectacle Othello a eu lieu le 5 octobre 1990, au Westvlaams Theater Antigone, à Kortrijk (Courtrai), pendant le directorat de Frank Van Erven, et dans la traduction de Bert Voeten.

Eclairage : Marc Cnops et Lode Denturck.

Maîtres d'armes : Rudi Delhem.

Assistant à la mise en scène : Wim Van Den Driessche.

\section{BIBLIOGRAPHIE}

Pour les chroniques de ce spectacle, voir Het Volk, 071091 ; Het Nieuwsblad, 071091 ; Gazet van Antwerpen, 081091 ; Het Laatste Nieuws, 091091 ; Knack, 161091 ; De Morgen, 161091, l'AvantScène, 0192. 